\title{
Effect of bur type and conditioning on the surface and interface of dentine
}

\author{
J. A. BARROS*, S. I. MYAKI ${ }^{\dagger}$, J. E. NÖR* \& M. C. PETERS* *Department of Cariology, Restorative \\ Sciences and Endodontics, University of Michigan, Ann Arbor, MI, USA and ${ }^{\dagger}$ Department of Pediatrics, UNESP-SJC, São José dos Campos, SP, \\ Brasil
}

SUMMARY The purpose of this in vitro study was to evaluate the surface and resin-dentine interface characteristics of permanent tooth dentine cut with diamond or carbide burs and treated with phosphoric acid (PA) or an acidic conditioner. Labial surfaces of permanent incisors were prepared into dentine with high-speed carbide or diamond burs and divided into two halves. Phosphoric acid 36\% was applied on one half and non-rinse conditioner (NRC) was applied on the other half. Ten randomly selected scanning electron microscopy (SEM) fields from each specimen $(n=15)$ were evaluated. Occlusal surfaces of third molars were divided in two halves for evaluation of the resin-dentine interface. The halves were randomly assigned to one of each conditioner and restored with Prime $\boldsymbol{\sigma}$ Bond NT/Spectrum. Ten specimens were analysed by SEM to evaluate hybrid layer formation and interfacial seal. We observed that surfaces prepared with carbide bur presented less residual smear plugs $(P<0.05)$ than surfaces prepared with diamond burs. Surfaces conditioned with NRC, which is a smear layer modifier, presented more residual smear plugs than surfaces conditioned with PA $(P<0.05)$. Treatment with $P A$ resulted in more sealed interfaces than specimens treated with NRC.Within the limitations of this study the results showed that carbide burs leave a surface that is more conducive to bonding than diamond burs. KEYWORDS: non-rinse conditioner, smear layer, resin/ dentine interface

Accepted for publication 30 January 2005

\section{Introduction}

The clinical outcome of dental restorations bonded to dentine is dependent upon the hybridization of adhesive resins into partially demineralized dentine, which provides stable adhesion. The literature has shown the importance of removal or modification of the smear layer for the development of a hybrid layer to obtain optimal adhesion $(1,2)$. The smear layer is created whenever a hand or rotary instrument is used to eliminate tooth tissue or surface layer. The debris produced by the instrumentation covers the dentine surface and obliterates the dentine tubules (3). Some studies have demonstrated that the thickness of the smear layer is affected by various factors such as type of bur, the use of water spray, and speed of rotation (4). It is also known that coarse diamond burs produce a thicker smear layer than carbide burs (5-7). The presence of a smear layer decreases dentine permeability and affects the outcome of bonded restorations (1).

Dentine bonding is enhanced when the smear layer is completely removed or modified exposing intertubular and peritubular dentine (8). Phosphoric acid (PA) is an etchant used not only to open the dentinal tubules, but also to demineralize the surface of the intertubular dentine and expose the dentine's collagen network $(9,10)$. After the dentine surface is demineralized by the etchant, the infiltration of adhesive resin into the collagen fibres creates micro mechanical retention. Although, previous studies have shown that the resin tags did not seem to improve the final bond strength (11), recent studies proved the hybrid layer to extend 

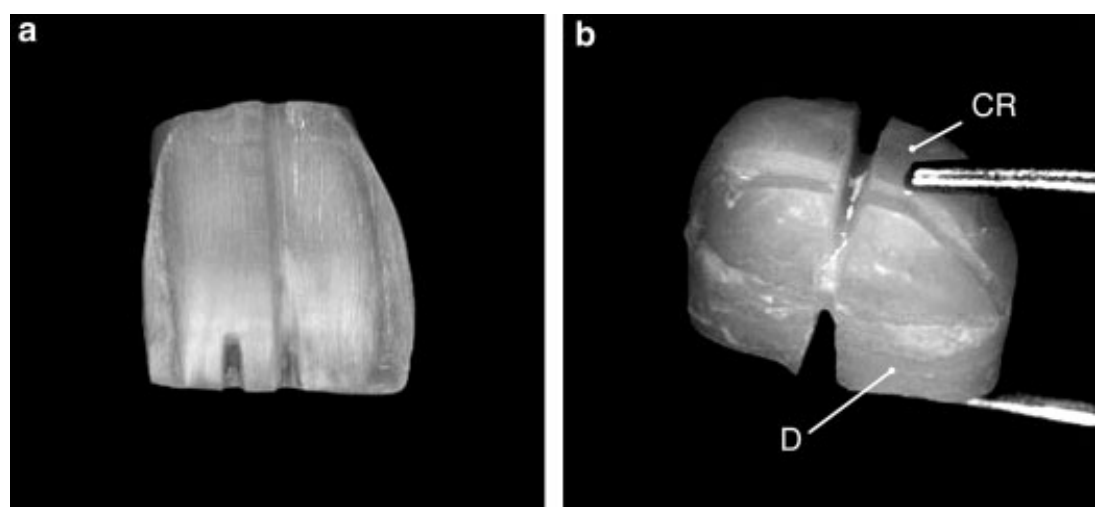

Fig. 1. (a) Specimen prepared with carbide bur, showing a groove dividing the tooth into two areas for application of non-rinse conditioner (NRC) and phosphoric acid (PA). Note the identification notch to distinguish the NRC side from the PA side. (b) Specimen being prepared for fracture. Note the grooves on the composite side and on the dentine side (CR: composite resin, D: dentine).

into the tubule orifice walls $(1,8,12)$. Because of this it may be important not only to remove the debris from the intertubular dentine but also to remove or modify the smear plugs.

Dentine surface treatment prior to any bonding procedure is essential to achieve optimal adhesion. Most of the bonding systems use an acid to condition enamel and dentine. In order to facilitate clinical application, new adhesive systems have been introduced to the market. Non-rinse conditioner (NRC)*, a self-etching system, is composed of two unsaturated dicarboxylic acids (maleic and itaconic) dissolved in water. Maleic acid acts as a conditioning agent, while itaconic acid behaves as a priming agent with the ability of copolymerizing with the Prime $\&$ Bond NT (13). This new material is claimed to modify the smear layer for creation of a good surface for adhesion.

The purpose of this in vitro study was to evaluate the effect of bur type and acidic conditioner on dentine surface and resin-dentine interface. The exposed dentine was restored to investigate the formation of hybrid layer and seal at the resin-dentine interface. The dentine surface was prepared with diamond or carbide burs and after treatment with PA (smear layer removal) or NRC (smear layer modification) the surface micromorphology was evaluated. The hypothesis tested was that surface characteristics and quality of interfacial seal achieved are similar whether the tooth is prepared using carbide or diamond, and NRC or standard acid etching was applied.

\section{Materials and methods}

\section{Dentine surface}

Fifteen sound human central incisors were selected for this study. The extracted teeth were stored in a solution of $0 \cdot 2 \%$ sodium azide for up to 1 month. The labial surface of each central incisor was flattened until superficial dentine was exposed, using a high-speed diamond bur and copious water spray. With a highspeed diamond bur the crowns were separated from the roots just apical to the cementoenamel junction. The teeth were randomly divided into three groups of five specimens. In Group 1 the dentine surface was prepared with a carbide bur $\# 171^{\dagger}$ under abundant water spray. A groove was placed from incisal to cervical in order to divide the tooth in two halves for different surface conditioning treatment (Fig. la). The same procedure was done with Group 2, using a diamond bur \#847$018^{\dagger}$. In Group 3, one half of the teeth was finished with a carbide bur \#171 and the other half with a diamond bur \#847-018. An identification groove was placed on the side to be treated with NRC.

The two materials used for conditioning of the dentine surface were NRC* and 36\% PA gel (Conditioner $\left.36^{*}\right)$. The surface treatment was randomly assigned to mesial and distal. In Groups 1 and 2, one half of the dentine was conditioned with PA for $15 \mathrm{~s}$ and rinsed with water for $15 \mathrm{~s}$. Subsequently the other side was treated with NRC. After application, the NRC

${ }^{\dagger}$ Brasseler, Savannah, Georgia, USA. 
Fig. 2. Diagram showing the specimen preparation and the fracture procedure for the interface investigation (NRC, non-rinse conditioner; PA, phosphoric acid; CG, central groove).

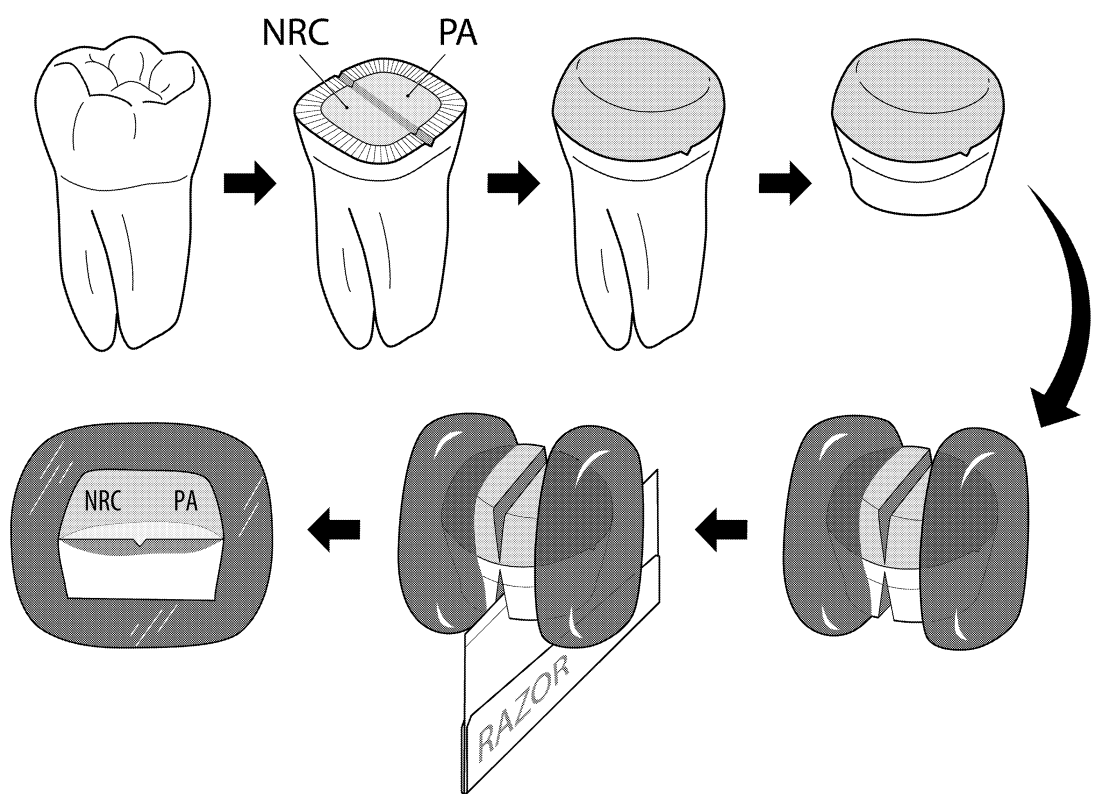

was left undisturbed for $20 \mathrm{~s}$ and gently blow-dried accordingly with manufacturer's instructions. An extra series of specimens in each bur group was included to analyse the removal of silica crystals present in the PA used. These specimens were etched for $15 \mathrm{~s}$ and rinsed for $30 \mathrm{~s}$ in order to observe under scanning electron microscopy (SEM) if the silica was removed. After dentine treatment, specimens were allowed to dry for $24 \mathrm{~h}$ under dust-free environmental conditions $\left(18^{\circ} \mathrm{C}\right.$, $40 \%$ humidity) followed by $24 \mathrm{~h}$ in a desiccator chamber $(14,15)$.

\section{Dentine interface}

Ten sound human molars were used for this experiment. All teeth were stored in $0 \cdot 2 \%$ sodium azide prior to the experiment. All teeth were flattened on the occlusal surface until dentine was exposed using a diamond bur with abundant water spray. These teeth were divided randomly in two groups, one with the surface treated with carbide bur \#171 and the other with diamond bur \#847-018. A central groove was prepared to separate the tooth into two areas. First, one side was treated with PA and the other half treated with NRC, as described above. Both groups were restored with Prime $\&$ Bond $\mathrm{NT}^{*}$ and Spectrum* following the manufacturer's recommendations. To minimize shrinkage stresses the composite resin was applied in increments until its height was about $4 \mathrm{~mm}$. All restorations were flat and parallel to the occlusal surface. To guide the fracture, a groove from mesial to distal was prepared in both sides (composite and dentine) of the specimen, using a taper diamond bur (Fig. lb). The specimens were embedded in acrylic resin to prevent the opening of the interface during fracturing procedures (Fig. 2). To evaluate the interface, the specimens were fractured using a singleedged blade oriented towards the dentine side. After the specimens were fractured, they were dehydrated for $24 \mathrm{~h}$ in a desiccator chamber.

\section{SEM evaluation}

All specimens were mounted on metal stubs and coated with gold using a sputter coater (Model S $150 \mathrm{~B}^{\ddagger}$ ). Each specimen was then analysed in a scanning electron microscope (Model $1000 \mathrm{~B}^{\S}$ ) with an accelerating voltage of $12 \mathrm{kV}$. The microscopic evaluation was done at a magnification of $1500 \times$ for the surface and $1000 \times$ for the interface.

For the surface evaluation, ten SEM fields from each half of the tooth surface were evaluated blindly by two investigators. The criterion used for evaluation of the smear layer was the removal of smear plugs. The smear layer was assessed as being 'removed' when $90 \%$ or more of the dentinal tubules where open (Fig. 3a), 'partially removed' (Fig. 3b), or 'present' when $10 \%$ or less dentinal tubules were exposed and open (Fig. 3c).

\footnotetext{
${ }^{\ddagger}$ Edwards Sputter Coater S150B, Edwards, USA.

${ }^{\S}$ Amray, Bedford, MA, USA.
} 

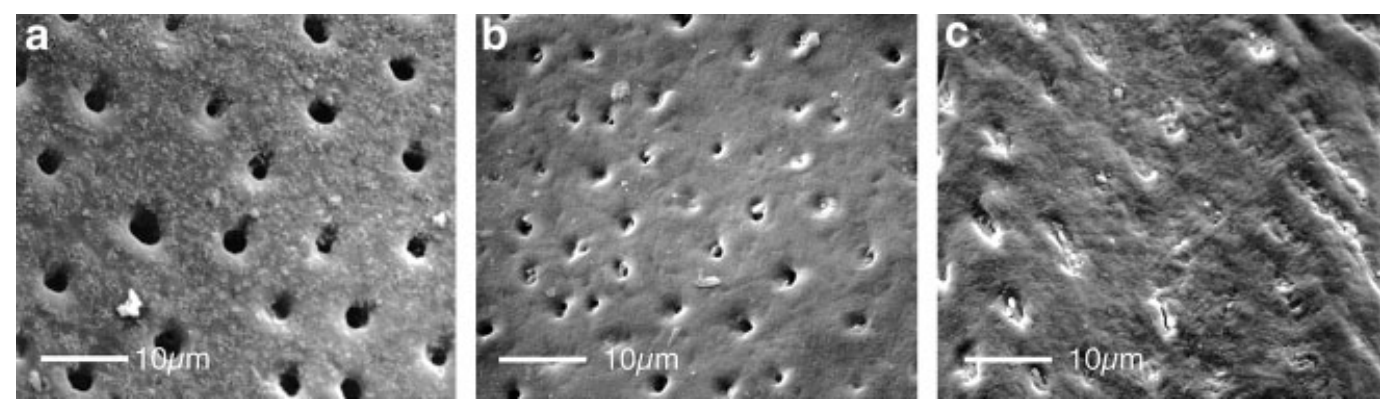

Fig. 3. Dentine surface prepared with: (a) carbide bur and $36 \%$ phosphoric acid (PA-15), showing a typical example of completely 'removed' smear layer; (b) diamond bur and 36\% phosphoric acid (PA-15), classified as 'partial removal' of smear layer; (c) diamond bur and non-rinse conditioner, classified as smear layer 'present' (original magnification $\times 1500$, bar represents $10 \mu \mathrm{m}$ ).

Table 1. Effect of surface treatment on the removal of smear plugs. The results are presented in percentage of presence, removal or partial removal of smear plugs

\begin{tabular}{|c|c|c|c|c|c|c|}
\hline \multirow[b]{2}{*}{ Smear plugs } & \multicolumn{3}{|l|}{ Carbide bur } & \multicolumn{3}{|l|}{ Diamond bur } \\
\hline & NRC & PA-15 & PA-30 & NRC & PA-15 & PA-30 \\
\hline Present & $8 \%(4 / 50)$ & $22 \%(11 / 50)$ & $16 \%(8 / 50)$ & $54 \%(27 / 50)$ & $32 \%(16 / 50)$ & $60 \%(30 / 50)$ \\
\hline Partially removed & $68 \%(34 / 50)$ & $50 \%(25 / 50)$ & $40 \%(20 / 50)$ & $44 \%(22 / 50)$ & $56 \%(28 / 50)$ & $32 \%(16 / 50)$ \\
\hline Removed & $24 \%(12 / 50)$ & $28 \%(14 / 50)$ & $44 \%(22 / 50)$ & $2 \%(1 / 50)$ & $12 \%(6 / 50)$ & $8 \%(4 / 50)$ \\
\hline
\end{tabular}

NRC, non-rinse conditioner; PA, phosphoric acid.

For the interface evaluation, each side of the specimen was analysed in order to determine if the interface was sealed without gaps showing the presence of a hybrid layer. A specimen was scored as being closed when the resin-dentine interface from the central groove to the mesial or distal side was completely sealed. The data were analysed by descriptive statistics, using chi-square comparison and Student's $t$-test to determine statistical differences at 0.05 per cent.

\section{Results}

The effect of the different surface treatments on the removal of the smear layer is shown in Table 1.

Representative surfaces are shown in Fig. 3. The data were presented in two groups: (A) the first group was treated with carbide bur and the surface conditioned in three ways: NRC, 36\% PA rinsed for $15 \mathrm{~s}$ (PA-15) (Fig. 3a) and 36\% PA with a 30 s rinse (PA-30); (B) the second group was treated exactly like the first one but using a diamond bur. In all cases the conditioned dentine surfaces showed a significant variability. Surfaces prepared with carbide bur presented less residual smear plugs $(P<0.05)$ after conditioning compared with surfaces cut with diamond burs. Surfaces condi- tioned with NRC presented more residual smear plugs $(P<0.05)$ than surfaces conditioned with 36\% PA. Each surface treated with PA showed a large amount of silica particles (Fig. 4). The presence of silica did not correlate with smear plug removal. The increase in rinsing time (30 s) did not enhance the removal of silica particles.

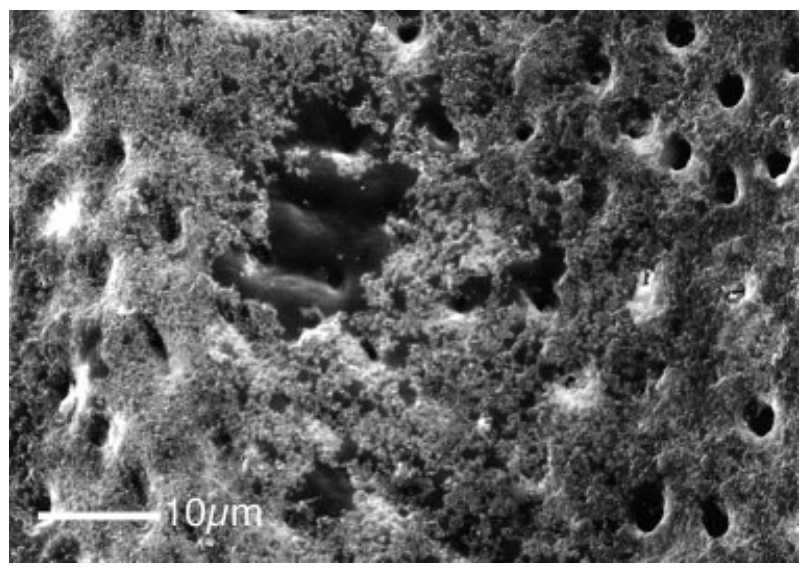

Fig. 4. This picture shows the amount of silica particles remaining on a dentine specimen after treatment with $36 \%$ phosphoric acid for $15 \mathrm{~s}$ followed by a $15 \mathrm{~s}$ rinse (original magnification $\times 1500$, bar represents $10 \mu \mathrm{m}$ ). 
Table 2. Qualitative assessment of the interfacial seal. Effect of surface treatment by bur type and conditioner on the quality of the resin-dentine interface

\begin{tabular}{|c|c|c|c|c|}
\hline \multirow[b]{2}{*}{ Interface } & \multicolumn{2}{|c|}{ Carbide bur } & \multicolumn{2}{|c|}{ Diamond bur } \\
\hline & PA & NRC & PA & NRC \\
\hline Opened & 1 & 4 & 2 & 3 \\
\hline Sealed & 4 & 1 & 3 & 2 \\
\hline Total & 5 & 5 & 5 & 5 \\
\hline
\end{tabular}

PA, phosphoric acid; NRC, non-rinse conditioner.

The effect of acidic conditioner and bur type on the resin/dentine interface is represented in Table 2. Evaluation of interface specimens prepared with carbide bur and PA showed $90 \%$ of the teeth evaluated with closed interfaces (Fig. 5). In the diamond group, $60 \%$ of the interfaces were closed. There was a significant variation in the NRC group. In the group of carbide specimens treated with NRC $(n=5)$ only one specimen showed a closed interface. Of the specimens treated with diamond and NRC $(n=5)$ two specimens presented a closed interface. In seven specimens with a sealed PA side, only three of seven NRC sides were sealed.

\section{Discussion}

A notable difference between the smear layer produced by carbide or diamond bur was observed in this study. Diamond burs produced a thick and uneven smear layer and the carbide burs produced a thin and evenly distributed smear layer confirming the results reported in the literature $(4,6,16)$. In many studies the $36 \%$ PA gel has proven to be the most reliable conditioner to clean the dentine surface prior to a bonding procedure (15, 17-19). An interesting finding was that 36\% PA did not completely remove the smear layer and smear plugs, especially when the specimens were treated with a diamond bur. The use of a diamond bur resulted in undulated surfaces and a thicker layer of debris was frequently accumulated at the deepest part of the undulations (Fig. 6). This variation in smear layer thickness may have contributed to uneven removal of smear layer by PA. Smoothness of the surface was

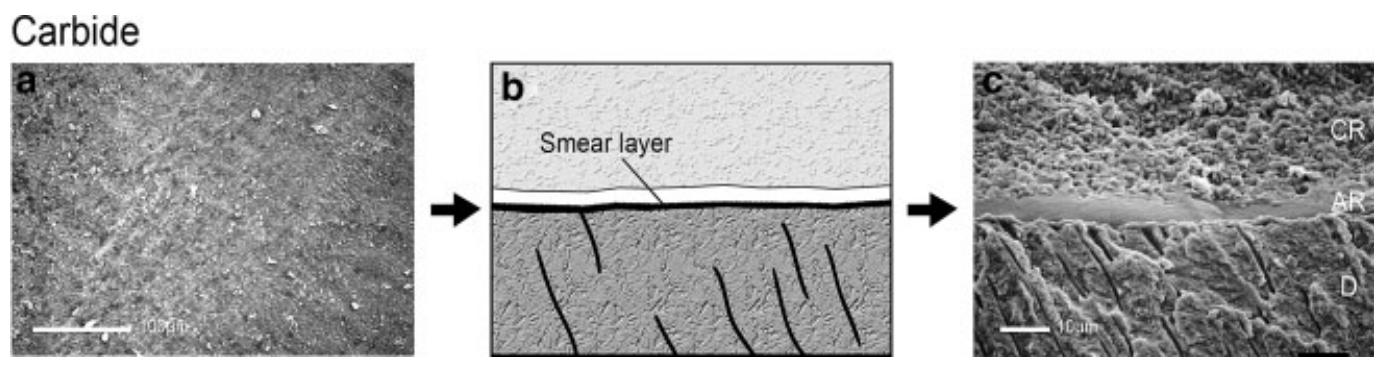

Fig. 5. Micromorphology of representative carbide treated surface and interface. (a) Surface showed the presence of a evenly distributed smear layer (original magnification $\times 100$, bar represents $100 \mu \mathrm{m}$ ); (b) graphical illustration of the deposit of smear layer on the surface caused by a carbide bur; (c) sealed resin/dentine interface in specimen treated with carbide bur and phosphoric acid (CR, composite resin; $\mathrm{AR}$, adhesive resin; $\mathrm{D}$, dentine; original magnification $\times 1000$, bar represents $10 \mu \mathrm{m}$ ).

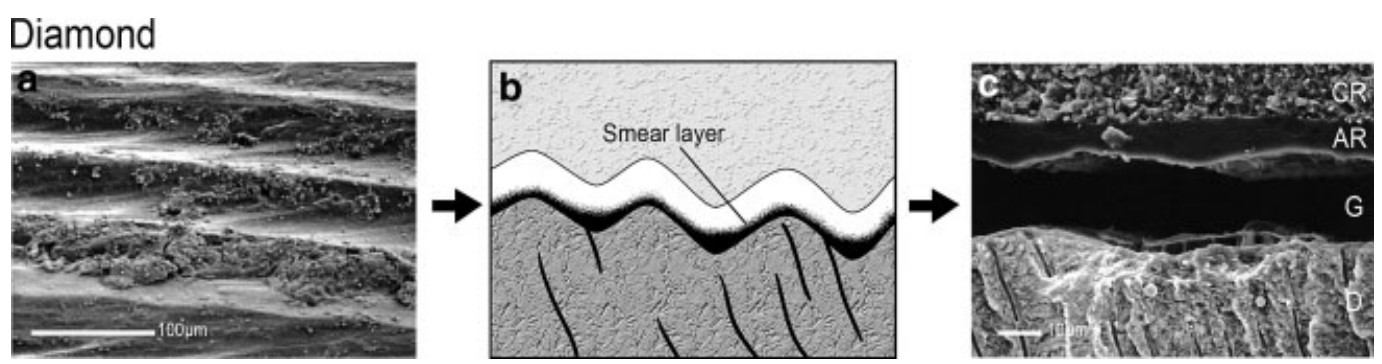

Fig. 6. Micromorphology of representative diamond treated surface and interface. (a) Surface showed the presence of thick and uneven smear layer (original magnification $\times 100$, bar represents $100 \mu \mathrm{m}$ ); (b) Illustration of the deposit of smear layer on the undulated surface caused by a diamond bur; (c) Open resin/dentine interface in specimen treated with diamond bur and non-rinse conditioner (CR, composite resin; AR, adhesive resin; G, gap; D, dentine; original magnification $\times 1000$, bar represents $10 \mu \mathrm{m}$ ). 
correlated with sealing of the interface. Cutting the dentine surface with regular grit diamond bur created a rough surface that resulted in more open resin-dentine interfaces. This finding corroborates with the work of Ogata et al. (16), who observed that a dentine surface cut with diamond bur and treated with a self-etching primer has areas with remnants of smear layer. In addition, Oliveira et al. (20) found that in general thick smear layers interfere with the adhesion capabilities of the self etching primer, suggesting that self-etching primers should be used with a surface preparation that creates a thin smear layer, like a carbide bur. On the contrary, Tani and Finger (21) stated that the differences in $\mathrm{pH}$ of the three adhesives tested in his study had were equally effective for a smear layer ranging between 0.9 and $2.7 \mu \mathrm{m}$ in thickness.

Most studies performed to investigate dentine surface treatment and bonding procedures use 600-grit SIC sandpaper to prepare a standardized surface $(18,22$, 23). However, this method produces a very flat and smooth surface and does not mimic the amount of smear layer created in clinical procedures with burs. In the present study a clinical scenario was simulated including burs that are commonly used for tooth preparation.

The specimens treated with $36 \%$ PA were coated with a significant amount of silica particles (Fig. 4). Therefore, five more specimens of carbide and diamond were prepared in order to observe if increased rinsing time to $30 \mathrm{~s}$ would improve silica removal from the surface. The results demonstrated that even after rinsing for $30 \mathrm{~s}$, the amount of silica particles did not decrease significantly. Perdigão et al. $(18,23)$ showed that silica did not affect bond strength. The authors also mentioned that the particles might prohibit deep demineralization of the intertubular dentine by the acid.

Çehreli and Altay (24) found that NRC is suitable as a conditioning agent on enamel and dentine in primary teeth. Baghdadi (25), however, concluded from his study on permanent and primary dentine that the mean shear bond strengths with NRC were remarkably lower than those obtained with PA. In the present study the dentine surfaces treated with NRC did not show complete removal of the smear layer and smear plugs were frequently observed. This is in accordance with the manufacturer's claim that this product does not remove the smear layer but modifies it. The NRC remnants left on the surface may copolymerize with the subsequently applied bonding agent and the dissolved smear layer eventually gets incorporated as filler particles (13). However, according to the literature $(1,2,8$, 12) the recommendation for bonding procedure is not only to dissolve the smear layer but also to remove the superficial part of the dentine, opening the dentinal tubules and intertubular dentine.

During specimen preparation for SEM, drying is necessary to achieve high vacuum. It is well known that preparation of biological specimens for SEM may generate artefactual changes $(18,26)$. Shrinkage frequently causes cracks and gap formation that does not exist in the original specimen. However, a study by Carvalho et al (27) showed that with all forms of specimen preparation, even taken precaution as fixation, ascending ethanol dehydration and critical point drying, shrinkage still occurs.

The present study protocol allowed the specimens to dry under environmental conditions $\left(18{ }^{\circ} \mathrm{C}, 40 \%\right.$ humidity). Therefore, the air-dried specimens showed some cracks and artefacts caused by the shrinkage of the demineralized dentinal tissue. Although the use of a more elaborate drying technique may have prevented some of the artefacts, the cracks in the specimens observed did not obscure the dentine surface morphology. The drying causes collapse of the demineralized collagen matrix. It is not likely that this would have severely masked patent dentinal tubules as the diameter of the tubule orifices is larger than the thickness of the collagen layer. Even though a different technique for specimen preparation was used the present study showed similar results as reported by Ogata et al. (16) and Oliveira et al. (20).

The self-etching conditioners have the ability to etch beyond the smear layer and demineralize the underlying intact dentine. All self-etching systems can bond reasonably well to dentine in the absence of patent dentinal tubules. Evaluation of resin/dentine interfaces is commonly done using dissolution of the underlying dentine substrate. However, the disadvantage of this dissolution method is that it is impossible to investigate the underlying dentine. Another option is to use a cross-sectioning technique, but this creates debris along the interface to be observed (12).

In the present study the interfacial integrity of fractured, air-dried specimens was examined under high vacuum. The advantage of this technique is the creation of an interface free of debris. On the contrary, the stresses caused by the fracture procedure can 
originate some damage and micro gaps. Alternative techniques using resin replicas backscatter SEM and environmental SEM all have their specific drawbacks (not allowing for evaluation of seal, gap or hybrid layer, low resolution). The fracture technique appeared to be the most suitable to investigate the interfacial seal. However, each of three parameters involved in the fracture technique (fracture, air-drying, high vacuum) can produce artefactual specimen cracking. To avoid confounding this study only included specimens that showed interfacial integrity on the non-experimental side. A closed interface at the adjacent side of the same tooth was used as pre-requisite in this study and served as internal control.

The positive results on the resin/dentine interface treated with carbide ( $90 \%$ sealed) are in line with our initial finding that the surface treated with carbide showed a more efficient and consistent removal of smear layer by PA. The interface investigation of the NRC conditioned specimens presented more open interfaces than specimens treated with PA. This finding contradicted the study by Ferrari et al. (28) who demonstrated that all dentine specimens treated with Prime $\&$ Bond NT and NRC presented a hybrid layer. It was observed that the thickness of the hybrid layer after application of NRC was thinner then when PA was applied. Also, the resin tags were shorter and of a narrower shape than those observed after treatment with PA. Recently, Tay and Pashley (29) observed that in permanent dentine NRC produced a hybrid layer where the smear layer was partially retained as part of the hybridized complex. However, two studies $(30,31)$ done to evaluate the microleakage and dentine-resin interface of NRC showed leakage on dentine in almost all specimens. In the present study, the NRC side of the specimens did not consistently present a hybrid layer. Whereas in the same specimen the internal control side (PA) was consistently sealed.

\section{Conclusions}

Based on the present study, it can be concluded that after conditioning with either PA or a NRC, the surfaces prepared with carbide bur presented significantly less residual smear plugs $(P=0 \cdot 02)$ than surfaces prepared with a diamond bur. Phosphoric acid treatment of a dentine surface cut with a diamond bur did not consistently remove the smear layer. No significant difference in residual smear plugs was found between surfaces conditioned with a smear layer modifier (NRC) and surfaces conditioned with PA, a smear layer remover. As expected, smear layer is more responsive to acid etching than to acidic primer. Specimens treated with PA resulted in more sealed interfaces than specimens treated with a NRC. In all cases, however, the conditioned dentine surfaces showed a large variability, underscoring the difficulty in achieving perfect bonding in clinical procedures. Within the limitations of this study the results showed that carbide burs leave a surface that is more conducive to bonding than diamond burs.

\section{References}

1. Eick JD, Gwinnett AJ, Pashley DH, Robinson SJ. Current concepts on adhesion to dentin. Crit Rev Oral Biol Med. 1997;8:306-335.

2. Blunk U. In: Roulet JF, Degrange M, eds. Adhesives: Principles and State of Art, Adhesion - the silent revolution in dentistry. Carol Stream, IL: Quintessence Publishing Co; 2000:29-44.

3. Eick JD, Wilko RA, Anderson CH. Scanning electron microscopy of cut tooth surfaces and identification of debris by use of the electron microprobe. J Dent Res. 1970;49:1359-1368.

4. Gwinnett AJ. Smear layer: morphological considerations. Oper Dent. 1984;3:2-12.

5. Brännström M, Glantz PO, Nordenvall KJ. The effect of some cleaning solutions on the morphology of dentin prepared in different ways: an in vivo study. ASDC J Dent Child. 1979;46:291-295.

6. Shortall AC. Cavity cleansers in restorative dentistry. Preliminary results from an in vitro scanning electron microscope study. Br Dent J. 1981;150:243-247.

7. Santini A, Mitchell S. A scanning electron microscopic study of the effect of Gluma CPS bonding system on dentinal smear layer produced by different bur types and rotational speeds and on the resin-dentin interface. Quintessence Int. 1998;29:737-747.

8. Perdigão J, Lopes M. Dentin Bonding - State of the Art 1999. Compend Contin Educ Dent. 1999;20:1151-1158, 11601162, 1164.

9. Pashley D. Dentin bonding: overview of the substrate with respect to adhesive material. J Esthet Dent. 1991;3:46-50.

10. Swift EJ, Perdigão J, Heymann HO. Bonding to enamel and dentin: a brief history and state of art. Quintessence Int. 1995;26:95-110.

11. Tagami J, Tao L, Pashley DH. Correlation among dentin depth, permeability, and bond strength of adhesive resins. Dent Mater. 1990;6:45-50.

12. Van Meerbeek B, Vargas M, Inoue S, Yoshida Y, Perdigão J, Lambrechts P, Vanherle G. Microscopy investigations. Techniques, results, limitations. Am J Dent. 2000;13:3D-18D.

13. NRC. Non-rinse conditioner technical manual. Konstanz: Dentsply DeTrey GmBH; 1998. 
14. Baba N, Taira Y, Matsumura H, Atsuta M. Surface treatment of dentin with Gluma and iron compounds for bonding indirect restorations. J Oral Rehabil. 2002;29:1052-1058.

15. Nör JE, Feigal RJ, Dennison JB, Edwards CA. Dentin Bonding: SEM comparison of the dentin surface in primary and permanent teeth. Pediatr Dent. 1997;19:246-252.

16. Ogata M, Harada N, Yamaguchi S, Nakajima M, Tagami J. Effect of self-etching primer vs phosphoric acid etchant on bonding to bur-prepared dentin. Oper Dent. 2002;27:447-454.

17. Nör JE, Feigal RJ, Dennison JB, Edwards CA. Dentin Bonding: SEM comparison of the resin-dentin interface in primary and permanent teeth. J Dent Res. 1996;75:1396-1403.

18. Perdigão J, Lambrechts $P$, Meerbeek BV, Tomé AR, Vanherle G, Lopes AB. Morphological field emission - SEM study of the effect of six phosphoric acid etching agents on human dentin. Dent Mater. 1996;12:262-271.

19. Ayad M. Effects of rotatory instrumentation and different etchants on removal of smear layer on human dentin. J Prosth Dent. 2001;85:67-72.

20. Oliveira SSA, Pugach MK, Hilton JF, Watanabe LG, Marshall SJ, Marshall GW Jr. The influence of the dentin smear layer on adhesion: a self-etching primer vs. a total-etch system. Dent Mat. 2003;19:758-767.

21. Tani C, Finger WJ. Effect of smear layer thickness on bond strength mediated by three all-in-one self-etching priming adhesives. J Adhes Dent. 2002;4:283-289.

22. Rontani RM, Ducatti CH, Garcia-Godoy F, De Goes MF. Effect of etching agent on dentinal adhesive interface in primary teeth. J Clin Pediatr Dent. 2000;24:205-209.

23. Perdigão J, Denehy GE, Swift EJ Jr. Silica contamination of etched dentin and enamel surfaces: a scanning electron microscopic and bond strength study. Quintessence Int. 1994;25:327-333.

24. Çehreli ZC, Altay N. Etching effect of 17\% EDTA and a nonrinse conditioner (NRC) on primary enamel and dentin. Am J Dent. 2000;13:64-68.

25. Baghdadi ZD. Bond strength of Dyract AP compomer material to dentin of permanent and primary molars: phosphoric acid versus non-rinse conditioner. J Dent Child. 2003;70:145-152.

26. Nation JL. A new method using hexamethyldisilazane for preparation of soft insect tissues for scanning electron microscopy. Stain Technol. 1983;58:347-351.

27. Carvalho RM, Yoshiyama M, Brewer PD, Pashley DH. Dimensional changes of demineralized human dentine during preparation for scanning electron microscopy. Arch Oral Biol. 1996;41:379-386.

28. Ferrari M, Mannocci F, Kugel G, Garcia-Godoy F. Standardized microscopic evaluation of the bonding mechanism of NRC/Prime \& Bond NT. Am J Dent. 1999;12:77-83.

29. Tay FR, Pashley DH. Aggressiveness of contemporary selfetching systems. I: Depth of penetration beyond dentin smear layers. Dent Mater. 2001;17:296-308.

30. Moodley D, Grobler SR, Rossouw RJ, Oberholzer TG, Patel N. In vitro evaluation of two adhesive systems used with compomer filling materials. Int Dent J. 2000;50:400-406.

31. Li H, Burrow MF, Tyas MJ. Nanoleakage patterns of four dentin bonding systems. Dent Mater. 2000;16:48-56.

Correspondence: Dr Mathilde C. Peters, Department of Cariology, Restorative Sciences and Endodontics, University of Michigan at 1011 N. University, D2361, Ann Arbor, MI 48109-1078, USA. E-mail: mcpete@umich.edu 